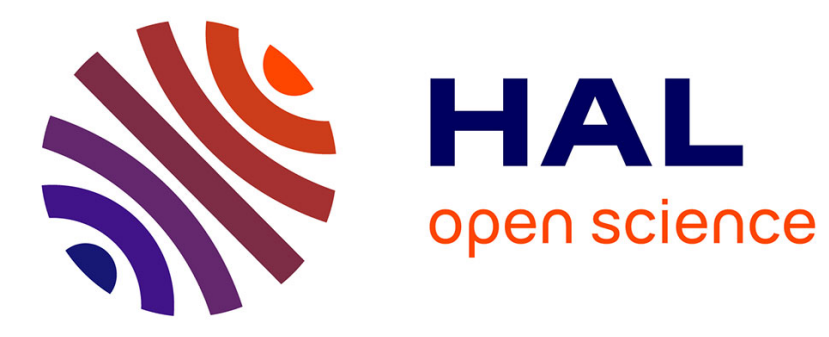

\title{
A local sensor for joint temperature and velocity measurements in turbulent flows
}

Julien Salort, Éléonore Rusaouën, Laurent Robert, Ronald Du Puits, Alice

Loesch, Olivier Pirotte, Philippe-Emmanuel Roche, Bernard Castaing,

Francesca Chillà

\section{To cite this version:}

Julien Salort, Éléonore Rusaouën, Laurent Robert, Ronald Du Puits, Alice Loesch, et al.. A local sensor for joint temperature and velocity measurements in turbulent flows. Review of Scientific Instruments, 2018, 89 (1), pp.015005. 10.1063/1.4989430 . hal-01667711

\section{HAL Id: hal-01667711 \\ https://hal.science/hal-01667711}

Submitted on 19 Dec 2017

HAL is a multi-disciplinary open access archive for the deposit and dissemination of scientific research documents, whether they are published or not. The documents may come from teaching and research institutions in France or abroad, or from public or private research centers.
L'archive ouverte pluridisciplinaire HAL, est destinée au dépôt et à la diffusion de documents scientifiques de niveau recherche, publiés ou non, émanant des établissements d'enseignement et de recherche français ou étrangers, des laboratoires publics ou privés. 


\title{
A local sensor for joint temperature and velocity measurements in turbulent flows
}

\author{
Julien Salort ${ }^{1}$, Éléonore Rusaouën ${ }^{2}$, Laurent Robert ${ }^{3}$, Ronald du Puits ${ }^{4}$, Alice Loesch ${ }^{4}$, \\ Olivier Pirotte $^{5}$, Philippe-E. Roche ${ }^{2}$, Bernard Castaing ${ }^{1,6}$, Francesca Chillà ${ }^{1}$
}

December 19, 2017

${ }^{1}$ Univ Lyon, Ens de Lyon, Univ Claude Bernard, CNRS, Laboratoire de Physique, F-69342 Lyon, France

${ }^{2}$ Univ. Grenoble Alpes, Inst NEEL, F-38042 Grenoble, France and CNRS, Inst NEEL, F-38042 Grenoble, France

${ }^{3}$ Femto-ST, UMR 6174, F-25030 Besançon, France

${ }^{4}$ Institute of Thermodynamics and Fluid Mechanics, Technische Universitaet Ilmenau, POB 100565, 98684 Ilmenau, Germany

${ }^{5}$ CERN, CH-1211 Geneva 23, Switzerland

${ }^{6}$ Univ. Grenoble Alpes, CNRS, Grenoble INP, LEGI, F-38000 Grenoble, France

\begin{abstract}
We present the principle for a micro-sensor aimed at measuring local correlations of turbulent velocity and temperature. The operating principle is versatile and can be adapted for various types of flow. It is based on a micro-machined cantilever, on the tip of which a platinum resistor is patterned. The deflection of the cantilever yields an estimate for the local velocity, and the impedance of the platinum an estimate for the local temperature. The velocity measurement is tested in two turbulent jets: one with air at room temperature which allows to compare with well-known calibrated reference anemometers, and another one in the GReC jet at CERN with cryogenic gaseous helium which allows a much larger range of resolved turbulent scales. The recording of temperature fluctuations is tested in the Barrel of Ilmenau which provides a controlled turbulent thermal flow in air. Measurements in the wake of a heated or cooled cylinder demonstrate the capability of the sensor to display the cross correlation between temperature and velocity correctly.
\end{abstract}

\section{Introduction}

\subsection{Turbulent velocity fluctuations}

The investigation of well resolved local Eulerian fluctuations has proved to be a fruitful approach to gather insights on turbulent flows. Local velocity, in particular, has been extensively studied in experimental homogeneous and isotropic turbulent flows. A very general feature of those flows is that a wide range of scales is involved, from the forcing scale down to the dissipation scale [1. As the forcing is increased, the range of scales gets larger. In laboratory flows, where the forcing scale cannot be made arbitrarily large, this means that the dissipation scale gets small. This prompted the need for ever faster and smaller local sensors.

One of the most successful approach is hot-wire anemometry [2]. Over the last three decades, it has triggered numerous discussions and led to the development of dedicated statistical tools and models [3, 4, 5]. In particular, it has allowed to produce well-resolved data for the study of intermittency in turbulence [6]. Hot-wire anemometers are still actively researched today, in particular for non conventional fluids, such as superfluid helium [7]. New design are investigated: fully micro-machined hot-wires [8, 9] are now approaching the few microns resolution of the smallest reported hot-wires [10, 11].

Despite this success, hot-wire anemometers also have shortcomings. We detail two situations more particularly: (i) the case of flows where changes in local flow direction may occur [12, and (ii) the case of thermally-inhomogeneous flows where warm or cold fluid parcels could significantly bias the signal. Indeed, hot-wire anemometers are based on the measurement of the heat-transfer efficiency from the wire to the surrounding fluid. In the case of isothermal flow, this efficiency depends only on forced convection, and therefore on the absolute value of the flow velocity. It is therefore intrinsically unable to detect a change of velocity direction. In the case of non-isothermal flows, it is hard to differentiate the passing of a cold fluid parcel and the passing of a faster fluid parcel. There have been attempts 
to tackle both problems in specific situations: (i) multiple wires can be used to infer changes in the flow direction [13, but only up to a maximum angle, or alternatively, the hot-wire can be complemented by a direction sensor [14], and (ii) models can be used to compensate for the temperature fluctuations, provided that a local temperature sensor is available 15 .

An alternative approach has been successfully proposed ten years ago by Barth, et al. [16. It is based on the atomic force microscope technique where cantilevers are used to detect extremely small forces. A micro-patterned cantilever is inserted inside the flow, its deflection yields an estimate for the local velocity. In the original setup from Barth, et al., the deflection is measured with optical means and the invasiveness of the optical system makes it possible to measure in one flow direction only. The measurement method has been recently improved to get two velocity components by measuring both the bending and the twisting of the cantilever [17. In addition, in the case of non-isothermal flows, light can be scattered by the optical index gradients, so this principle of detection is not immune to temperature fluctuations. Yet, they demonstrated the high sensitivity and resolution of this measurement method, as well as its applicability to water flows where hot-wire anemometers do not perform well, because they cannot be heated as much, and bubbles may always nucleate on the heated wire.

Five years ago, we have extended this technique of cantilever-based anemometry to the case of low temperature liquid helium flows [18. We then proposed a method based on a superconducting micro-resonator patterned onto the cantilevered beam. This is made possible by the progress in micro-machining techniques. The advantage of the superconducting micro-resonator was its high sensibility. The main shortcoming is that it requires a high enough quality factor and no spurious sensibility on the kinetic inductance, both of which are obtained when the phonon density gets small, i.e. at very low temperatures, well below the material superconducting critical temperature. In practice, this sensor works well below $2 \mathrm{~K}$, and is therefore well suited to study superfluid helium flows. However, it cannot work at higher temperature a-priori.

\subsection{Turbulent scalar dynamics}

Despite the academic success of homogeneous and isotropic model flows, both in terms of experiments and modelling, these are seldom well suited to describe actual natural or industrial systems. In many systems, it is necessary to take into account scalar fields, e.g. temperature or salt in density-driven flows, such as thermal convection, or pollutants and catalysts in the industrial flows. The scalar field can be passive, i.e. simply advected by the flow, or active, i.e. locally forcing the flow.

For example, the understanding of the dynamics of passive scalars in turbulent flows is important to predict the dispersion of pollutants. Theoretical efforts have been made to model the situation [19, 20]. For these predictions to be validated experimentally, one has to measure local scalar and velocity correlations. One experimental caveat is that the smallest scale of such a flow, called the Batchelor scale, $\beta$, is given by,

$$
\beta=\eta S c^{-1 / 2},
$$

where $\eta$ is the dissipative scale of turbulence (Kolmogorov scale), and $S c=\nu / D$ is the Schmidt number, where $\nu$ is the fluid viscosity and $D$ the scalar diffusivity. In the case of salted water, the Schmidt number is of order 1000, hence the Batchelor scale is nearly 30 times smaller than the Kolmogorov scale. This means that the sensors have to be smaller than in the case of isothermal flows.

One traditionnal method consists in setting up velocity measurement and temperature measurement, independantly of each other, e.g. fast cold wires combined with either Laser-Doppler-Anemometry [21], or Particle-Image-Velocimetry [22]. However, achieving accurate synchronisation and ensuring that the measurement points precisely match is not straightforward. We present a novel sensor design, aimed at measuring jointly the local velocity and the local value of a scalar field. This is done using a cantilever anemometer onto which additional material is sputtered and patterned. In all generality, this additional sensing element should be chosen to match the needs of a particular flow, such as a temperature sensitive material to be used as a thermometer, or a set of electrodes to be used as conductometer.

In this paper, we focus on the case of the joint temperature and velocity measurements. The prototype is a cantilever onto which both a strain gauge and a temperature-sensitive resistor have been patterned. The ultimate aim of this sensor is to grant access to the local temperature and velocity cross-correlations, and thus to the local turbulent heat flux. The strain gauge may be less sensitive than the optical technique used by Barth, et al., and the superconducting micro-resonator we previously used. However, it is much less invasive than the former and can operate on a wide range of temperatures, both at room temperatures and down to cryogenic helium temperatures. 


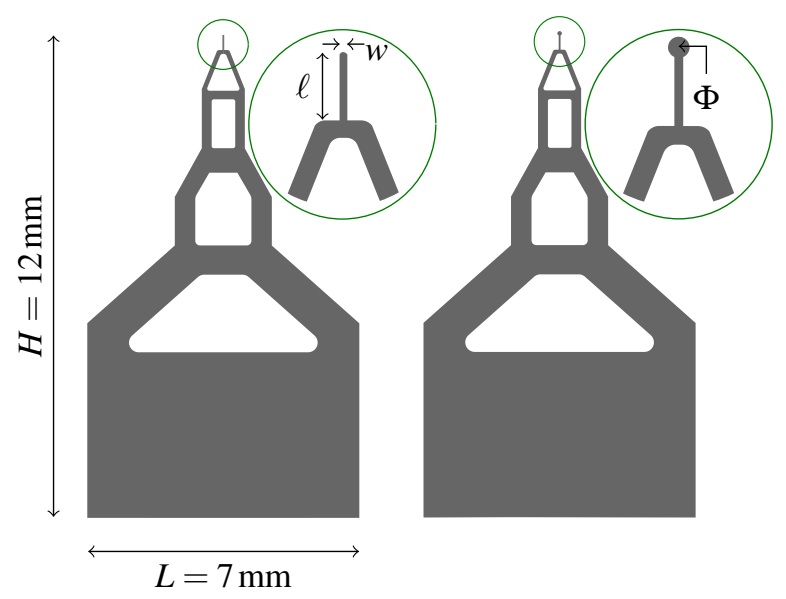

Figure 1: Sketch of the joint temperature and velocity micro-sensor with main dimensions. Left: "Straight" cantilever. Right: "Racket-shaped" cantilever. $\ell=375 \mu \mathrm{m} . w=35 \mu \mathrm{m} . \Phi=100 \mu \mathrm{m}$.

\section{Sensor \& Fabrication process}

The sensor consists in a $1.2 \mu \mathrm{m}$-thick silicon oxide cantilever onto a $390 \mu \mathrm{m}$-thick bulk silicon support and bearing arms. The bearing arms are $120 \mu \mathrm{m}$-wide at the base of the cantilever, and get larger and larger while drawing away from it, as can be seen in figure 1 . This is a compromise between robustness and invasiveness. Future sensors may use narrower arms to reduce invasiveness further.

The fabrication starts from the thermal oxidation of double-side polished $\langle 100\rangle$ bulk silicon wafers. This allows fine control of the thickness of the silicon oxide that will become the cantilever. The thermometer circuit, strain gauge circuit and the tracks are then realised by iterating the same steps: (i) oxygen plasma cleaning; (ii) spin-coating of photosensitive resist (Ti09XR from MicroChemicals in our case); (iii) photo-lithography of the pattern; (iv) evaporation or sputtering of a thin film; and (v) lift-off and cleaning.

The sensors presented in this paper use (i) $1200 \AA$-thick evaporated platinum on a $100 \AA$ chromium thin layer as the resistance thermometer; (ii) $(1850 \pm 350) \AA$-thick sputtered Constantan on a chromium thin layer as a strain gauge material; and (iii) $1650 \AA$-thick evaporated gold on a $100 \AA$ chromium thin layer for tracks (see figure 2 . The thin layers of chromium are used to promote the adhesion of the thin films on the substrate. Platinum was chosen for its stability and well-known temperature dependence. Constantan was chosen for its documented low dependence on temperature, to avoid spurious temperature-driven signal on the strain bridge. As the sputtered layer composition may deviate from the one of the commercial $99.5 \%$-pure target we used, its final composition was measured by EDS-X. The ratio in mass are, within $\pm 0.5 \%$ uncertainty, $49 \%$ copper, $49 \%$ nickel, $1.1 \%$ manganese. Finally, gold was chosen for the tracks for its stability and softness.

Earlier versions of the sensor used larger sputtered $1200 \AA$-thick Nichrome as strain gauge, sputtered $1400 \AA$-thick platinum as resistance thermometer, and evaporated $2000 \AA$-thick gold for tracks, all onto a thin $400 \AA$ chromium layer to promote adhesion. These earlier sensors had significant residual stress, which yielded an angle of order $40^{\circ}$, instead of a horizontal beam. This was caused by the use of sputtering instead of evaporation, and a larger gauge pattern. In some flows with a large mean velocity, this might be seen as an advantage, as the mean velocity may deflect the cantilever back to horizontal.

The silicon dioxide is patterned by using a photolithography step and a BHF etching.

The final step is the deep reactive ion etching of the bulk silicon via the bottom side to form the bearing arms and release the silicon dioxide cantilever. As a mask for this dry etching, we used a patterned $7 \mu \mathrm{m}$-thick photoresist (AZ9260 from MicroChemicals) and then the bottom side of the wafer is etched in a SPTS Rapier module using Bosch switched processing to achieve vertical silicon side walls. 


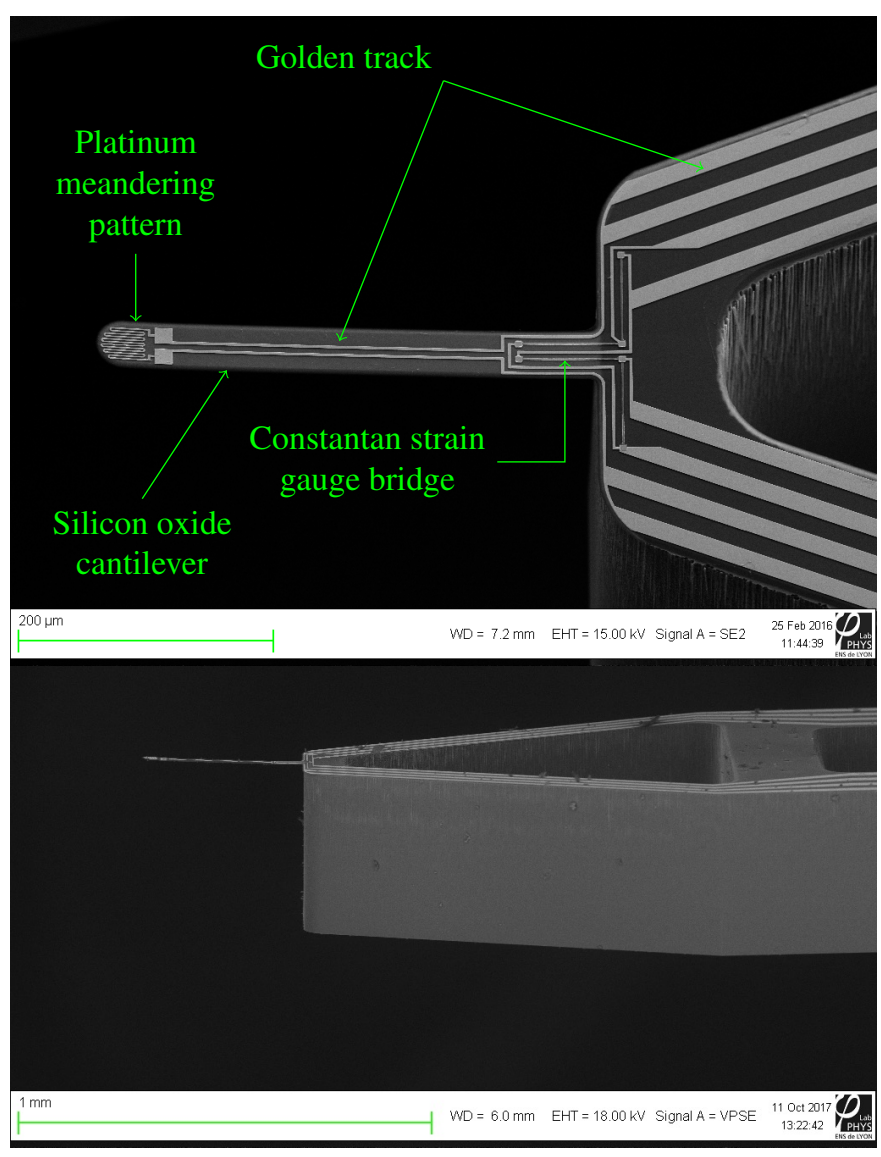

Figure 2: Scanning Electron Microscope pictures of a micro-structured cantilever, viewed from the top and from the side. 


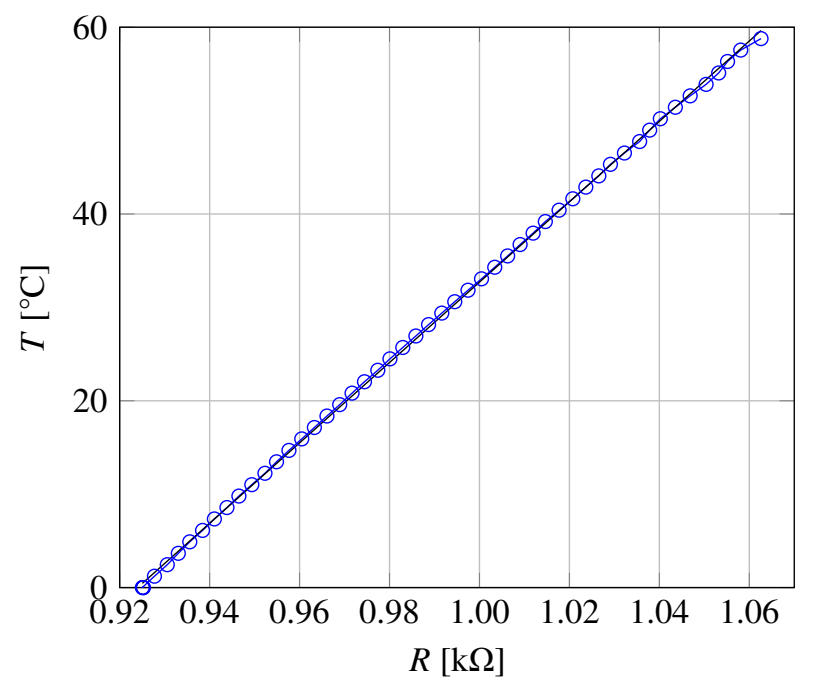

Figure 3: Calibration of the platinum resistance thermometer embedded on the structured cantilever sensor. Solid line is the linear fit, $T=\alpha R+T_{0}$ with $\alpha=0.4304{ }^{\circ} \mathrm{C} \Omega^{-1}$ and $T_{0}=-397.5{ }^{\circ} \mathrm{C}$.

\section{Sensor calibration}

\subsection{Calibration of the platinum thermometer}

The resistance of the platinum meandering circuit is measured with the 4-wire method using a Hewlett-Packard HP34401a multimeter on its $100 \mathrm{k} \Omega$ range $\left(10 \mu \mathrm{A}\right.$ measuring current) with $6{ }^{1 / 2}$-digits resolution. Higher currents would lead to a measurable self-heating of the resistance thermometer. The sensor is installed on a bulk copper cylinder, itself inserted in the ethylene-glycol bath of a Lauda RP845 chiller. The bath temperature was swept up and down and the resistance measurements obtained for each sweep collapse within the experimental uncertainty. This ensures that the temperature stabilisation time was sufficient, and that there were no hysteresis of any sort.

The measurements shown in figure 3 evidence a linear relationship. The derived sensibility, $\sigma$, is

$$
\sigma=\frac{1}{R} \frac{\partial R}{\partial T}=2.52 \times 10^{-3}{ }^{\circ} \mathrm{C}^{-1},
$$

slightly less sensitive than commercial bulk platinum resistors which have a sensibility of $3.91 \times 10^{-3}{ }^{\circ} \mathrm{C}^{-1}$. This is not highly surprising as material properties in thin layers are known to possibly deviate from those of bulk materials. This is also much less sensitive than semiconductor-based thermistors, but platinum layers have the advantage of long-term stability.

The sensor response time can be compared to other state-of-the-art micro-thermometers, such as micro-thermistors, fast cold-wires, or micro-thermocouples. One simple way to quantify the response time is to submit the sensor to a temperature step, and measure the response time $\tau_{70}$ at which the sensor has achieved $70 \%$ of the total jump. The response time is shorter when the sensor is placed in a flow, so this estimate is an upper bound. As shown in figure 4 the present sensor response time, $\tau_{70}$, is found below $1 \mathrm{~ms}$, for a temperature step of nearly $20^{\circ} \mathrm{C}$, without external flow.

It lies within similar range as the dedicated in-house micro-thermocouples developed by Munzel \& Kittel 23 for which temperature steps up to $250 \mathrm{~Hz}$ were performed, and the micro-machined T-NSTAP cold-wire from Princeton University 24. This is more than 100 times faster than one of the smallest commercial micro-thermistors, such as the one used by du Puits, et al., $130 \mu \mathrm{m}$ in diameter and $330 \mu \mathrm{m}$ in length, for which $\tau_{70}$ was found [25] between $140 \mathrm{~ms}$ without external flow and $90 \mathrm{~ms}$ with an external flow velocity $1 \mathrm{~m} / \mathrm{s}$.

Compared to micro-thermistors, our sensor may reflect the fluid small-scale temperature more accurately thanks to the low thermal inertia of the $1.2 \mu \mathrm{m}$-thick silicon oxide layer that it is made of, while still benefiting from a relatively large heat transmitting surface $(100 \mu \mathrm{m}$ diameter $)$, and the low spurious heat transport across the contacting wires. The volume, and therefore presumably the heat capacity, of the cantilever tip is nearly 1000 times smaller than those of micro-thermistors. 


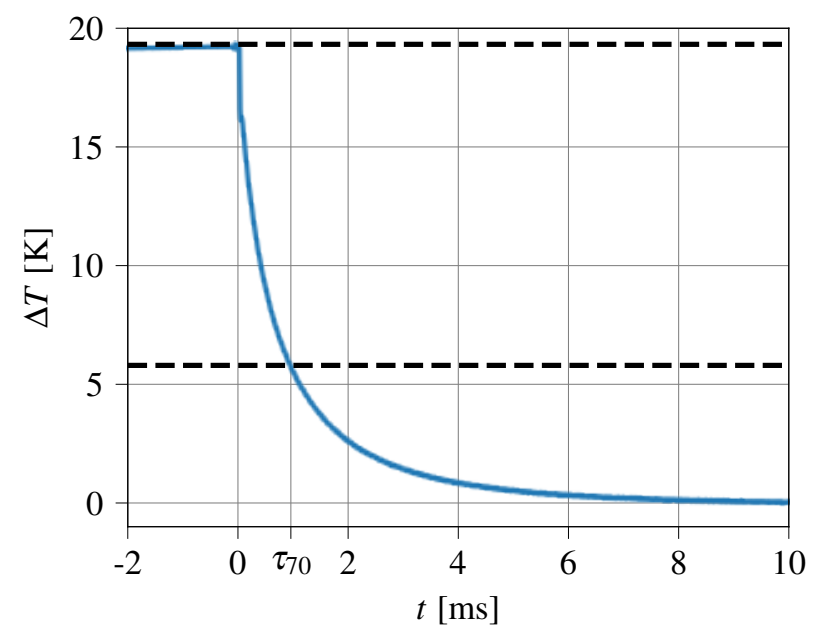

Figure 4: Sensor platinum layer response to a temperature step in air without external flow. The heating power is $66 \mu \mathrm{W}$. The response time, $\tau_{70}$ at which time the sensor has achieved $70 \%$ of the total jump, is $960 \mu s$.

Because the gauge bridge takes most of the width of the cantilever near the base of the cantilever, it was not possible, in the present design, to get a fully 4-wire connection to the platinum meander: there is a portion of the golden tracks which will contribute to the measured impedance (see figure 2), e.g. the measured impedance, $R$, can be written

$$
R(T)=R_{P t}(T)+R_{\text {gold }}(T, \delta \ell)
$$

where $R_{P t}(T)$ is the impedance of the platinum meander which depends on temperature only, and $R_{\text {gold }}(T, \delta \ell)$ is the impedance of the 2-wire portion of the golden tracks which may also depend on the relative elongation of the cantilever beam.

The golden tracks have a length of order $830 \mu \mathrm{m}$, a width $2.5 \mu \mathrm{m}$, and a thickness $160 \mathrm{~nm}$. The platinum meander has a length $340 \mu \mathrm{m}$, width $1.25 \mu \mathrm{m}$ and a thickness $120 \mathrm{~nm}$. Gold is nearly five time more conductive than platinum. This allows to estimate the contribution of the golden tracks,

$$
\frac{R_{\text {gold }}}{R_{P t}}=r \sim 0.2,
$$

which is small but not negligible. The contribution of the golden tracks to the measured impedence hence partly explains why the measured sensitivity is smaller than the reference sensitivity of platinum.

Additionnally, the golden tracks themselves may act as a spurious strain gauge, and yield unwanted velocity signal on the thermometer. However, only the base of the cantilever gets elongated, which represents less than a quarter of the total length of the tracks. Yet, let us derive an estimate of the typical spurious temperature error induced by strain on the golden tracks. The gauge factor of pure metals is of order 1, therefore

$$
\frac{\delta R_{\text {gold }}}{R_{\text {gold }}} \sim \frac{\delta \ell}{\ell} .
$$

Hence, the spurious variation of measured resistance caused by the relative elongation $\delta \ell / \ell$ is

$$
\delta R=R_{\text {gold }} \frac{\delta \ell}{\ell}=r R_{P t} \frac{\delta \ell}{\ell} .
$$

which can be rewritten using Eq. 2 .

$$
\delta T=\frac{\delta R}{\sigma R}=\frac{r}{(1+r) \sigma} \frac{\delta \ell}{\ell} .
$$

As shown in the next section, the typical relative elongation of the strain gauge is of order $\delta \ell / \ell \sim 10^{-4}$. This is a conservative upper bound for the golden tracks as a quarter of their length may actually be elongated, at most. Yet, using this estimate, Eq. 7 yields $\delta T \sim 6 \mathrm{mK}$, which is relatively small compared to the typical temperature fluctuations in room temperature Rayleigh-Bénard convection experiments. 


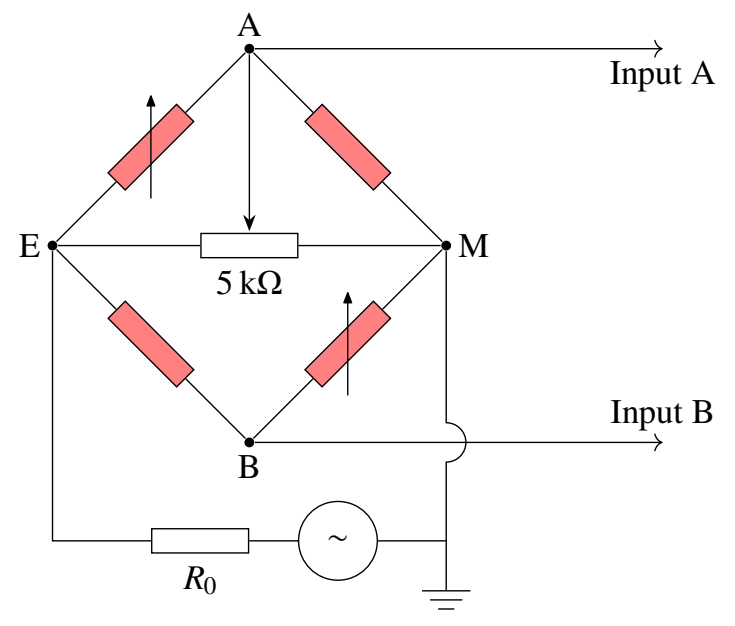

Figure 5: Sketch of the measurement method of the gauge bridge imbalance. The four resistors filled in red are the Constantan thin-film resistors patterned on the micro-system (typical resistance $450 \Omega$ ). The $5 \mathrm{k} \Omega$ potentiometer is added to compensate for the residual imbalance. The $R_{0}=27.1 \mathrm{k} \Omega$ resistor is chosen to tune the input current. The low frequency voltage generator, and the inputs A and B are those of the Stanford SR830 lock-in Amplifier. The voltage amplitude is $5 \mathrm{~V}$ at a frequency $27.52 \mathrm{kHz}$.

\subsection{Calibration of the strain gauge}

The relative elongation of the beam top surface caused by a uniform pressure load $P$ on this surface can be written

$$
\frac{\Delta \ell}{\ell} \propto \frac{P}{E} \frac{\ell^{2}}{e^{2}},
$$

where $E$ is the Young modulus of silicon oxide [18. The pressure load induced by the motion of the fluid impinging on the cantilever normally can be written

$$
P=\frac{1}{2} c_{d}(v) \rho_{f} v^{2},
$$

where $c_{d}(v)$ is the drag coefficient, $\rho_{f}$ the fluid density and $v$ the local velocity. Therefore, the voltage on the strain bridge, $U$, is expected to be

$$
U \propto \frac{1}{2} \rho_{f} \operatorname{sign}(v) c_{d}(v) v^{2} .
$$

To perform the calibration, a "straight" cantilever sensor, with dimensions shown in figure 1 is first placed inside an air jet at room temperature. The nozzle diameter is $1 \mathrm{~cm}$ and the distance between the nozzle and the sensor is $20 \mathrm{~cm}$. The wind velocity at this point was calibrated with a TSI hot-wire and a CTA-1750 electronics. It can be chosen between $0 \mathrm{~m} / \mathrm{s}$ and $8.5 \mathrm{~m} / \mathrm{s}$ by varying the power of the motor. The sensor can be turned upside-down to change the velocity direction. We use a Stanford SR830 lock-in amplifier to measure the bridge imbalance (see figure 5). The measurements are shown in figure 6

The sensor response appears to be fairly linear at small velocities and quadratic for larger velocities. This could be caused by the separation of the boundary layers near the probe. The calibration data is well fitted by a function of the form,

$$
U=a v+b_{ \pm} v^{2},
$$

where $b_{ \pm}$is $b_{+}$if $v>0$ and $b_{-}$if $v<0$. Because the geometry of the sensor is not symmetric in the thickness direction, the width of the cantilever being much smaller than that of the supporting arms (see figure 2), the response may not a-priori be similar for positive or negative velocities. That is why the values of $b_{+}$and $b_{-}$were free during the fit. Yet, they were found to have the same absolute value. Eq. 11 may thus be rewritten,

$$
U=a v-\operatorname{sign}(v) b v^{2},
$$

where $b=b_{-}=-b_{+}>0$. 


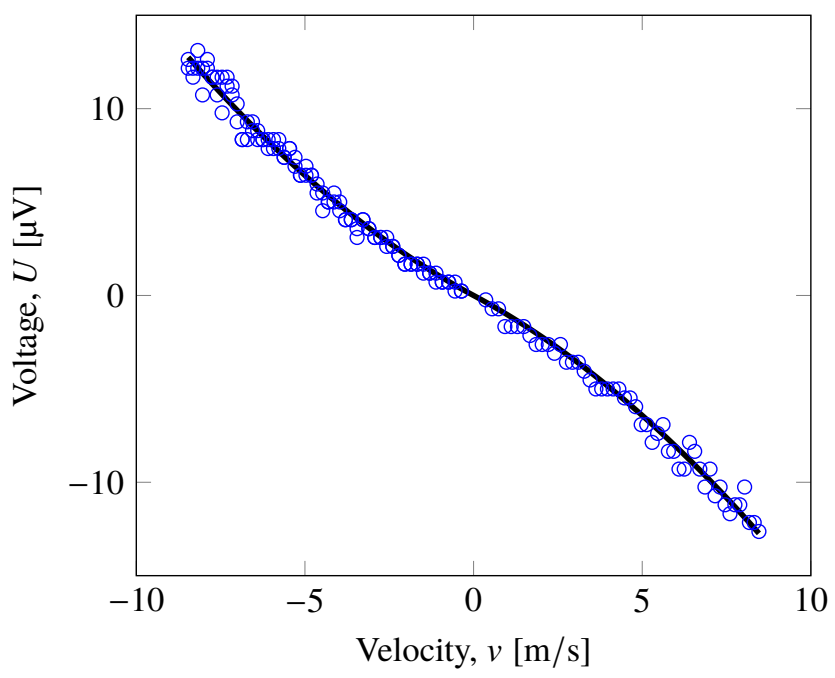

Figure 6: Calibration of the cantilever anemometer for flows in both directions. Solid line is a fit $U=a v+b_{ \pm} v^{2}$, with $a=-0.97 \mu \mathrm{Vm}^{-1} \mathrm{~s}, b_{-}=-b_{+}=6.4 \times 10^{-2} \mathrm{\mu Vm}^{-2} \mathrm{~s}^{2}$.

From this fit function, one may infer a typical threshold velocity, $v_{0}$, characteristic of the transition from the linear to the quadratic behaviour:

$$
v_{0}=\left|\frac{a}{b}\right| \sim 15 \mathrm{~m} / \mathrm{s}
$$

The local probe Reynolds number,

$$
R e_{p}=\frac{w v}{\nu},
$$

where $\nu=1.5 \times 10^{-5} \mathrm{~m}^{2} / \mathrm{s}$ is the kinematic viscosity of air, is $R e_{p}=34$ for $v=v_{0}$, which can be compared to typical transition Reynolds numbers, keeping in mind that the value would be larger if the length of the beam, $\ell$ were chosen as the typical probe scale instead of its width (or the racket diameter in the case of racket-shaped cantilevers). As a comparison [26], a cylinder of diameter $w$ would classically start exhibiting wakes at $R e_{p} \approx 30$ and its drag coefficient would be changing from a $c_{d} \sim 1 / R e_{p}$ behaviour to a constant value in the range between $R e_{p}=10$ and $R e_{p}=100$. The observed change of regime is thus consistent with the typical probe Reynolds numbers for which a change in the drag coefficient is expected.

One advantage of this sensor design is that it should allow velocity measurements over a wide range of working temperature, down to cryogenic conditions. The Constantan strain resistors have an impedance of order $450 \Omega$ at room temperature, and we measured variations smaller than $5 \%$ when cooling them down to cryogenic temperatures. We have tested the sensitivity of the strain bridge at cryogenic temperature with an early version of the sensor (using Nichrome as a strain gage, as mentioned previously) inserted in the GReC cryogenic gaseous helium round jet [11. Preliminary measurements in several systems were previously carried out and showed that Nichrome and Constantan have similar gauge factor. Therefore comparison between early Nichrome sensors or latest Constantan ones is possible.

The gas temperature was $6.0 \mathrm{~K}$ and density $11.1 \mathrm{~kg} / \mathrm{m}^{3}$. The mass flow rate could be chosen between 0 and $125 \mathrm{~g} / \mathrm{s}$ only, because the experiment was being refurbished. Higher flow rates should be possible in the future.

To allow comparison of the signals obtained in air and in cryogenic helium, the fluid properties must be taken into account, and the signal must be made dimensionless to account for the values of bridge polarization. We define, $s,($ in $\%)$, as

$$
s=100 \times \frac{U}{U_{0}},
$$

where $U$ is the imbalance bridge voltage, and $U_{0}$ the bridge polarization voltage. The obtained cantilever signal, $s$, is compared to the prediction of Eq. 10 in figure 7. To do that, an estimate of the drag coefficient, $c_{d}\left(R e_{p}\right)$, is required. Its exact value is not known. That is why we used the simple classical experimental values of the drag coefficient of a cylinder [26] of diameter $w$ as an approximation for the "straight" cantilever, and the drag coefficient of a circular plate [27] of diameter $\Phi$ as an approximation for the "racket-shaped" cantilever. 


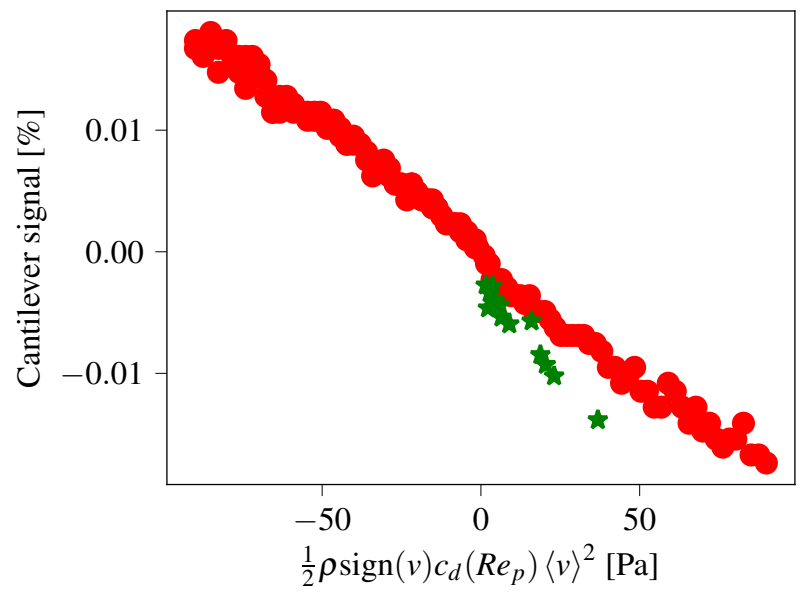

Figure 7: Comparison of the mean signal obtained in air with a "straight" cantilever (full red symbols) and in cryogenic gaseous helium with a "racket-shaped" cantilever (green stars). We used the drag coefficient of a circular cylinder [26] for $c_{d}(R e)$ for the "straight" cantilever, and the drag coefficient of a circular plate 27] for the "racket-shaped" cantilever.

The values from the air jet and from the cryogenic helium jet collapse fairly well (see figure 7), which shows that the sensor sensitivity is similar at room temperature and at cryogenic temperature. The small discrepancy can originate from the estimates of drag coefficients and from differences in strain gauge coefficients. For the "straight" cantilever, it would be also possible to use experimental drag coefficient values of a rectangular plate of aspect ratio 0.093 given by Hoerner [27, but the uncertainty is slightly larger in this range of Reynolds number and the discrepancy marginally larger.

Naturally, the probe Reynolds number, $R e_{p}$, is the main control parameter for the sensor behavior. Its typical value in the air jet is between 1 and 25, while the values in the GReC helium jet is between 260 and 1300. For this reason, only the air jet exhibits a range of quasi-linear voltage versus velocity relationship. In cryogenic helium, the calibration function is always quadratic because the drag coefficient, $C_{d}(R e)$, is nearly constant.

\subsection{Mechanical resonance frequency}

One possible limitation of cantilevers as anemometers is that their mechanical resonance frequency might lie within the range of hydrodynamical frequencies. In the case of parallelepiped cantilevers in vacuum, the flexion resonance frequency can be computed analytically [28]:

$$
f_{\mathrm{vac}, n}=\frac{1}{2 \pi} C_{n}^{2} \frac{\theta}{\ell^{2}} \sqrt{\frac{E}{12 \rho_{c}}},
$$

where $C_{n}$ are the roots of the equation,

$$
1+\cos C_{n} \cosh C_{n}=0,
$$

$\theta$ is the cantilever thickness, $\ell$ is its length, $\rho_{c}$ is the density of the cantilever and $E$ its Young modulus. In the following, we consider the fundamental flexion mode only because other modes, such as torsional modes, occur at higher frequency 29 .

We can derive the fundamental resonance frequency, $f_{\mathrm{vac}, 1}$, for the "straight" sensor using Eq. 16, with $C_{1}=1.875$, $E=70 \mathrm{GPa}$ and $\rho_{c}=2200 \mathrm{~kg} / \mathrm{m}^{3}$. It gives $f_{\mathrm{vac}, 1}=7.8 \mathrm{kHz}$. While this is higher than any frequency in natural convection flows, it might turn out to be a limitation for large Reynolds number flows.

However, one simple way to tackle the problem is to reduce the length of the beam. For a cantilever length of $160 \mu \mathrm{m}$ (the length of the Barth, et al. cantilever), Eq. 16 yields $f_{\mathrm{vac}, 1}=42.7 \mathrm{kHz}$. The choice of cantilever geometry is therefore a compromise between the sensitivity and the dynamical response of the sensor, and highly depends on the kind of flow that is considered. As an example, Eq. 16 yields $f_{\mathrm{vac}, 1}=99 \mathrm{kHz}$ for $140 \mu \mathrm{m} \times 40 \mu \mathrm{m} \times 1.6 \mu \mathrm{m}$ silicon cantilever (the Young modulus of silicon is nearly twice as large as the Young modulus of silicon oxide), which is consistent with the direct mechanical resonance measurements of Puczylowski, et al. [17. 
For the racket-shape cantilever, we expect the resonance frequency to be lower. Indeed, the beam is similar to a mass-spring system, with identical spring constant, but an additional mass, $\delta m$, due to the disk at the tip of the beam. The moment of inertia becomes

$$
I=I_{0}\left(1+\frac{3 \delta m}{m_{0}}\right)
$$

where $I_{0}$ and $m_{0}$ are the moment of inertial and the mass of the straight cantilever, and

$$
\frac{\delta m}{m_{0}}=\frac{\pi \Phi^{2}}{4 w \ell} .
$$

One might therefore roughly expect a correction,

$$
\frac{f_{\text {racket }}}{f_{\text {straight }}}=\left(1+\frac{3 \pi \Phi^{2}}{4 \ell w}\right)^{-1 / 2}=0.60
$$

and therefore a fundamental frequency in vacuum $f_{1}=4.7 \mathrm{kHz}$.

Additionally, the frequency response of the cantilever may be lowered further in fluids. The two main reasons are the effect of the fluid added mass, and the damping due to viscosity [30, 31. The frequency shift for cantilever resonance caused by viscous effects have been extensively studied as it impacts the cantilever thermal noise power spectrum [32, 33, 34]. This has indeed implications for use of cantilevers in AFM microscopes in liquid medium.

For a rectangular beam, the inviscid fluid model of $\mathrm{Chu}$ can be used to predict the cantilever resonance frequency in fluid 30

$$
\frac{f_{\text {fluid }}}{f_{\text {vac }}}=\left(1+\frac{\pi \rho_{f} w}{4 \rho_{c} \theta}\right)^{-1 / 2}
$$

where $\rho_{f}$ is the density of the fluid. This inviscid model is valid if the Reynolds number is large. The appropriate Reynolds number, $R e_{\omega}$ is 30

$$
R e_{\omega}=\frac{\pi f w^{2}}{2 \nu} .
$$

At $f=f_{\text {vac }}$, the value of $R e_{\omega}$ highly depends on the working fluid. It is $R e_{\omega} \sim 1$ for air at $25^{\circ} \mathrm{C}, R_{\omega} \sim 15$ for water at $25^{\circ} \mathrm{C}$, and $R e_{\omega} \sim 100 \gg 1$ for cryogenic gaseous helium at $6 \mathrm{~K}$ and $11.1 \mathrm{~kg} / \mathrm{m}^{3}$. In the following, we only consider fluid corrections in the case of helium because this prototype is not suitable for water yet, and the shift due to added mass and viscous effects in air are negligible.

In the helium case, the Reynolds number $R e_{\omega}$ is large, therefore only inertial effects must be taken into account. Combining Eqs. 20 and 21. and using $\Phi$ instead of $w$ for the typical length in the model of Chu, the fundamental frequency for the racket shape cantilever in gaseous cryogenic helium is $f_{1} \approx 4 \mathrm{kHz}$. The resonance frequency of the racket-shape cantilever will therefore be a limitation in cryogenic turbulent flows but it can be improved by reducing the length $\ell$.

\section{In-flow validations}

\subsection{Velocity fluctuations in turbulent jets}

The cantilever anemometer is placed in the same air jet discussed in section 3.2 The distance to the nozzle tip $(36 \mathrm{~cm})$ is such that the flow starts exhibiting turbulent features, and the mean velocity remains sufficiently high. We use the electronics shown in figure 5 with a lock-in time constant of $30 \mu$ s. The demodulated output signal is recorded with a National Instruments PXI-4462 acquisition card, with a sampling frequency $50 \mathrm{kHz}$. The corresponding power spectra, shown in figure 8 , have been computed with the Welch method with $2^{13}$ points per segment.

As illustrated in figure 8 the power spectra are fairly similar to those obtained using a hot-wire, except that the signal-to-noise ratio is lower. Indeed, the main shortcoming of the present prototype is a relatively low sensibility. This may improve in the future, if more sensitive strain gauge materials are used instead of Constantan, or if the geometry is changed to allow for higher excitation voltage. The hot-wire and cantilever signals deviate a little at low frequency: this may come from slightly different forcing (both signals have been acquired separately), or from slow fluctuations of the flow temperature which would yield spurious signal on the hot-wire but not on the cantilever. In the inertial range, the spectrum slope is close to the Kolmogorov $f^{-5 / 3}$ power law, though slightly less steep. This could be caused by a bottleneck phenomenon [35, or by geometric details. Indeed, our spectra are compatible with 


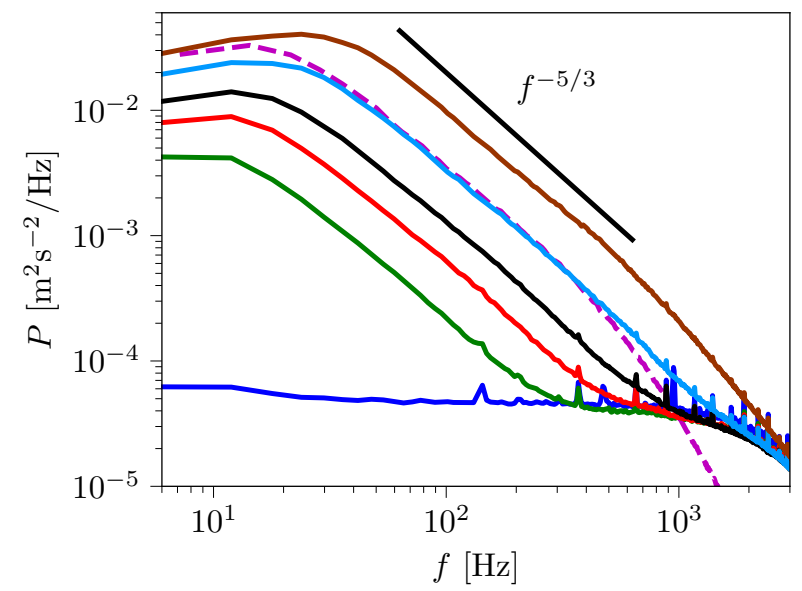

Figure 8: Solid lines: power spectra of the cantilever signal in the air jet. The sensor is $36 \mathrm{~cm}$ downstream the jet nozzle. From bottom to top, the mean velocity is $0 \mathrm{~m} / \mathrm{s}, 2.9 \mathrm{~m} / \mathrm{s}, 3.4 \mathrm{~m} / \mathrm{s}, 4.0 \mathrm{~m} / \mathrm{s}, 5.2 \mathrm{~m} / \mathrm{s}$ and $7.4 \mathrm{~m} / \mathrm{s}$. Dashed magenta line is an example of hot-wire measurement at the same position for similar mean velocity $(5.2 \mathrm{~m} / \mathrm{s})$.

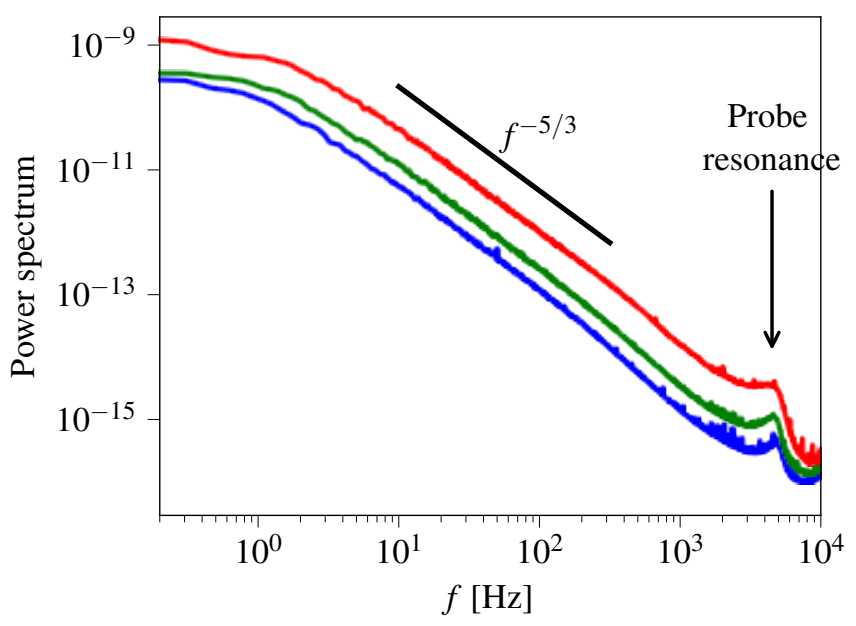

Figure 9: Cantilever signal power spectra in the GReC cryogenic gaseous helium round jet. From bottom to top: $77 \mathrm{~g} / \mathrm{s}, 88.5 \mathrm{~g} / \mathrm{s}$ and $125 \mathrm{~g} / \mathrm{s}$. 


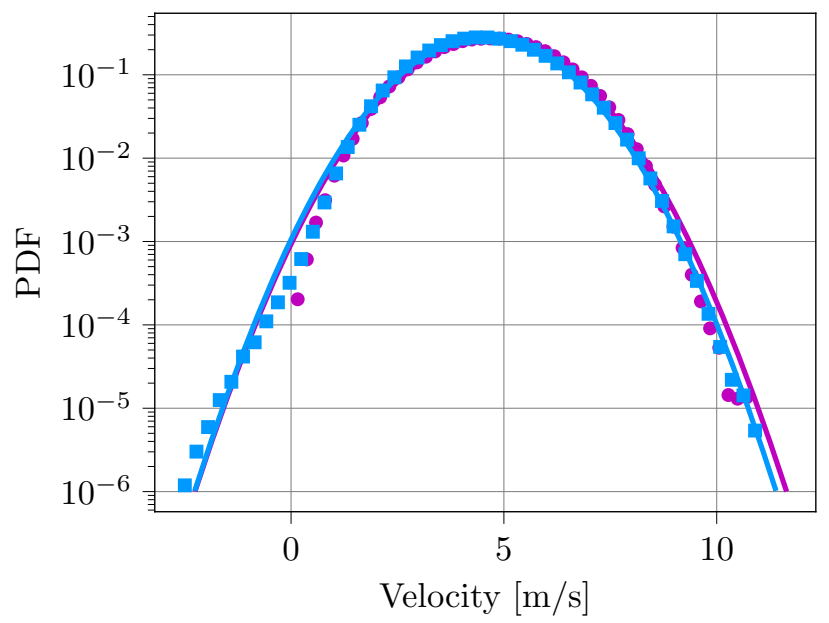

Figure 10: Velocity probability density function for the cantilever signal (black squares) and the hot-wire signal (magenta circles) at nearly similar mean velocity $\langle v\rangle=4 \mathrm{~m} / \mathrm{s}$. Solid lines are Gaussian distribution.

the results of Mi \& Antonia in a turbulent open round jet for similar distance to the nozzle and similar Reynolds numbers [36] where spectra were found with slopes between -1.5 at the center of the jet, and -1.7 near the edges.

The signal fluctuations have also been recorded in the GReC cryogenic helium round jet, which allows to investigate the sensor response at higher Reynolds number, and on a wider range of frequencies. Unfortunately, there were no cryogenic hot-wire available during that experiment that we could compare against.

The spectra are shown in figure 9 and evidence two decades of $f^{-5 / 3}$ scaling. The wide range of frequencies in the flow and the lower noise level allows to evidence a peak near $4 \mathrm{kHz}$, which is in fair agreement with the expected mechanical resonance frequency of the cantilever beam. The high-frequency cut-off due to the cantilever dimensions is expected at $\langle v\rangle / \ell \sim 6 \mathrm{kHz}$ (for $\langle v\rangle \sim 1.2 \mathrm{~m} / \mathrm{s}$ ). It is higher than the resonance frequency, and not visible on the spectra because it is filtered out by the anti-aliasing filter of the acquisition card.

Though the dynamics is better in the cryogenic jet than in the room temperature air jet, the latter is better suited to detailed analysis because well documented reference anemometers are available (TSI hot-wires), which allows accurate calibration. In addition, the GReC experiment was still in its early refurbishment process at the time of this experiment, and we could not guarantee that the flow remained stationary over the duration of the recordings.

One of the advantages of cantilever anemometry over hot-wires is that negative velocity values can be measured. Though the turbulent velocity fluctuations need not be exactly Gaussian [37, they are usually assumed to be nearly so (and symmetric). Inside the jet, one may thus expect rare events of negative velocity. The PDF of the cantilever and hot-wire signals are shown in figure 10. For small velocities, the hot-wire PDF shows a clear cut-off, as the signal cannot possibly be negative. The cantilever signal exhibits negative values.

The deviation from Gaussianity can be assessed by the flatness $F_{4}$ of the velocity fluctuations, also sometimes referred to as kurtosis, and defined as

$$
F_{4}=\frac{\left\langle(v-\langle v\rangle)^{4}\right\rangle}{\left\langle(v-\langle v\rangle)^{2}\right\rangle^{2}} .
$$

Experimental estimates of the turbulent velocity flatness have been reported by Noullez, et al., using a presumably unbiased optical method [38. They found $F_{4}=2.85$, slightly below the Gaussian value $\left(F_{4}=3\right)$. Other published estimates have been obtained with numerical simulations [39, 40, or using hot-wires: $F_{4}=2.66$ was found in the atmosphere [4], and values ranging from sub-Gaussian to Gaussian and to hyper-Gaussian inside a turbulent grid flow [42].

The signals shown in figure 10 yield two estimates for the turbulent jet in air: $F_{4}=2.80$ from the cantilever signal, and $F_{4}=2.72$ from the hot-wire signal. Indeed, hot-wire signals in such high intensity turbulent flow tend to underestimated flatness values because of the low-velocity cut-off. The signal obtained with the cantilever is in fair agreement with the value of Noullez, et al.

Yet, the cantilever PDF in figure 10 slightly deviates from a symmetric distribution around zero-velocity. One 


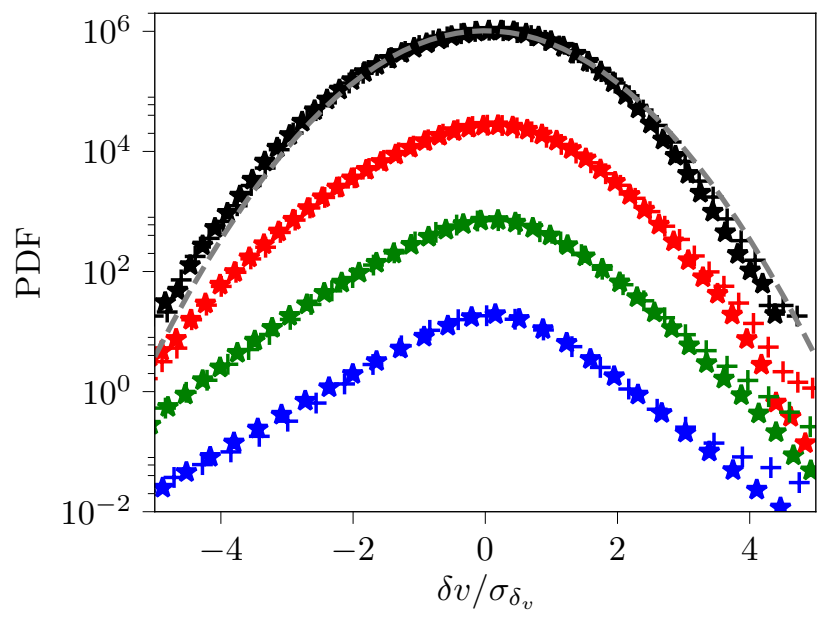

Figure 11: Probability density function of the longitudinal velocity increments measured with hot-wire (stars) and the cantilever (plus). From bottom to top, and assuming the hypothesis of Taylor frozen turbulence, $\delta r=1 \mathrm{~mm}$, $\delta r=3 \mathrm{~mm}, \delta r=10 \mathrm{~mm}$ and $\delta r=30 \mathrm{~mm}$. For reference, the gray dashed line is the Gaussian distribution. Curves are arbitrarily offset vertically to improve readability.

likely reason is that the signal is very weak for low velocities, which makes it hard to measure accurately. The accuracy of the calibration function may also be less reliable in this region.

Finally, one important feature of Eulerian turbulence is the statistics of the longitudinal velocity increments, $\delta v\left(r_{0} ; \delta r\right)$,

$$
\delta v\left(r_{0} ; \delta r\right)=v\left(r_{0}+\delta r\right)-v\left(r_{0}\right),
$$

where $r_{0}$ is the probe position. The longitudinal distance $\delta r$ can be related to the time offset $\tau$ assuming Taylor frozen turbulence hypothesis,

$$
\delta r=\langle v\rangle \tau .
$$

To validate the velocity signals further, the probability density function of the longitudinal velocity increments, obtained in the air jet from the cantilever sensor and the reference hot-wires, are shown in figure 11. The integral scale is $2 \mathrm{~cm}$. Though the smallest resolved scale is only of order a tenth of the integral scale, the deviation from Gaussianity is clearly visible on both signals. The PDF computed from the cantilever and from the hot-wire fairly agree in the range of scale that the prototype can resolve: clearly non Gaussian at $\delta r=1 \mathrm{~mm}$, but fairly Gaussian, though expectedly slightly skewed, for $\delta r=30 \mathrm{~mm}$.

\subsection{Temperature measurements in turbulent convection}

Because the metal layers are not insulated, the present prototype is not suited for use in water. For this reason, we have installed it in the Barrel of Ilmenau, a large thermal convection facility in Ilmenau which uses air as the working fluid 43. More precisely, we placed the cantilever micro-sensor at the center of a convection cell made from Plexiglas walls inserted into the Barrel, close to the bottom plate.

The setup is identical to the one described by Liot, et al. 44]: a $2.50 \mathrm{~m} \times 2.50 \mathrm{~m}$ rectangular cell with $0.50 \mathrm{~cm}$ thick Plexiglas walls, between two horizontal aluminum plates. The bottom plate temperature is $55.0^{\circ} \mathrm{C}$, the top plate temperature is $15.0^{\circ} \mathrm{C}$, which yields a Rayleigh number,

$$
R a=\frac{g \alpha \Delta T H^{3}}{\nu \kappa}=4.7 \times 10^{10},
$$

and a Nusselt number,

$$
N u=\frac{Q H}{\lambda S \Delta T}=247,
$$

where $g$ is the gravitation acceleration, $\alpha$ the thermal expansion coefficient, $\Delta T=40^{\circ} \mathrm{C}$ is the temperature difference, $H=2.50 \mathrm{~m}$ is the height of the convection cell, $Q$ is the power applied on the heating plate, $S$ is the surface area of 


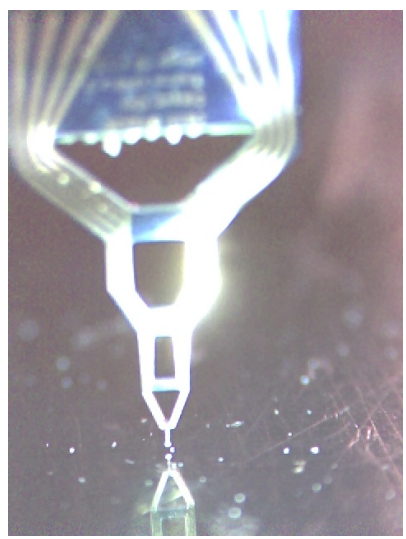

Figure 12: Hand-microscope picture of the cantilever probe as close to $z=0$ as possible. The aluminum plate at $z=0$ acts as a mirror, so that the sensor reflection can be seen. The actual distance to the bottom plate is less than $200 \mu \mathrm{m}$.

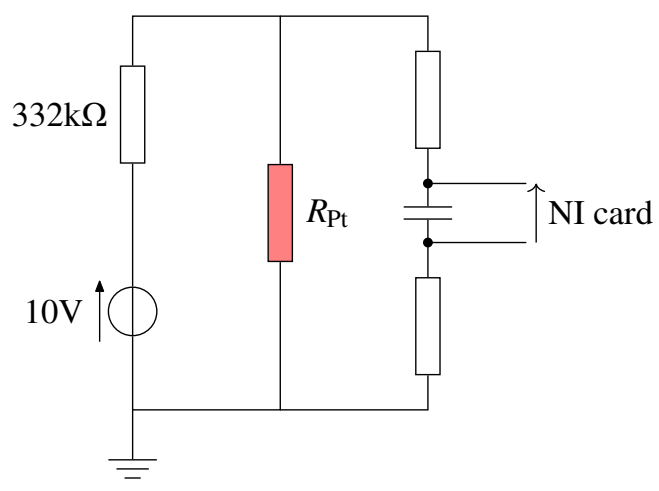

Figure 13: Electronic diagram of the platinum resistor 4-wire fluctuation measurements. The resistor filled in red is the platinum thin film on the micro-system. The first order $R C$ filter cut-off frequency is $338 \mathrm{~Hz}$. The average resistance of the platinum thin film is $R_{\mathrm{Pt}}=1 \mathrm{k} \Omega$.

the plates, $\nu$ is the kinetic viscosity, $\kappa=\lambda / \rho c_{p}$ is the thermal diffusivity and $\lambda$ is the thermal conductivity. More information on turbulent Rayleigh-Bénard convection can be found in the review by Chillà and Schumacher 45 .

The sensor is installed near the center of the bottom plate, on a movable vertical rod, which can be adjusted with a step motor. The origin, $z=0$, is set by imaging the sensor with a hand-microscope to help positioning it as close to the plate as possible, as illustrated in figure 12 At this location, Particle Image Velocimetry (PIV) analysis has previously shown that the boundary layer displacement thickness is $9 \mathrm{~mm}$ and the viscous sublayer is of order $1.7 \mathrm{~mm}$. The typical plume size is expected to be of the same order of magnitude, thus much larger than the sensor typical size.

Unfortunately, this prototype is not sensitive enough to measure velocity accurately in these conditions. However, the setup can be used to validate the temperature fluctuation data from the platinum thin layer. One advantage of this probe over other temperature sensors is that it has a negligible thermal inertia, and is located at the tip of a non-conductive silicon oxide rod. The reader might refer to the work of Gauthier, et al. for a full discussion on the relevance of local thermometer response time in turbulent convection [46].

The platinum resistance fluctuations are recorded using the electronic diagram shown in figure 13 . The $10 \mathrm{~V}$ voltage is supplied by a battery. The output current is $30 \mu \mathrm{A}$. The results are shown in figure 14 .

The histograms are consistent with reference temperature fluctuation data in turbulent Rayleigh-Bénard convection [47]: the temperature histogram is nearly symmetrical inside the thermal boundary layer (recording at $z=190 \mu \mathrm{m}$ in the figure), and in the bulk well outside the boundary layers (recording at $z=148 \mathrm{~mm}$ in the figure). Outside, but close to the boundary layer $(z=21 \mathrm{~mm}$ in the figure), the histogram is strongly skewed due to the advection of thermal plumes. They are parcels of hot fluid (near the bottom plate), or cold fluid (near to top plate). 

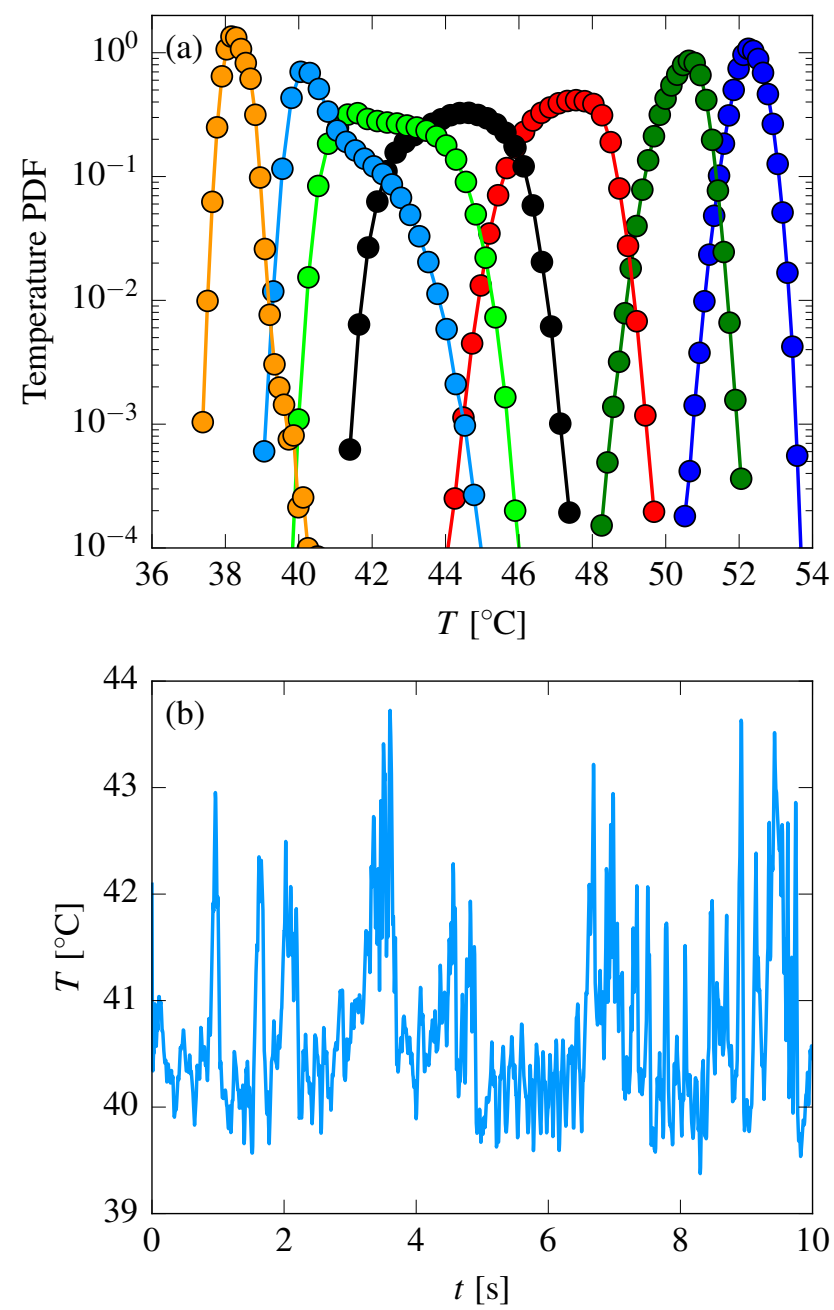

Figure 14: (a) Temperature histograms recorded by the micro-sensor thin-film platinum resistor. From left to right: $z=148 \mathrm{~mm}$ (orange), $z=21 \mathrm{~mm}$ (cyan), $z=10 \mathrm{~mm}$ (green), $z=4.0 \mathrm{~mm}$ (black), $z=1.3 \mathrm{~mm}$ (red), $z=410 \mu \mathrm{m}$ (dark green), $z=190 \mu \mathrm{m}$ (blue). (b) Sample of temperature recording at $z=21 \mathrm{~mm}$.

Events of plumes crossing the sensor are clearly visible on the signal sample in figure $14 \mathrm{~b}$. At this location, the background temperature is $40^{\circ} \mathrm{C}$, and plumes are recorded with temperatures up to $43.5^{\circ} \mathrm{C}$.

We can therefore conclude that the thin-film platinum resistor at the tip of the cantilever micro-system is sensitive and fast enough to resolve the temperature fluctuations in turbulent thermal convection in air. The results are comparable to those obtained with micro-thermistors [47, 48. One advantage of this design is that it can easily be scaled up in an array of micro-thermometers, that will grant access to local temperature correlations. It is also more stable as platinum does not drift. Compared to micro-thermocouples, which are also known to give good results in turbulent thermal convection [49], this sensor geometry ensures a better exchange with the fluid (larger contact surface) with reduced spurious conduction to the sensor frame (negligible conduction in silicon oxide).

\subsection{Joint temperature and velocity measurements behind a cylinder}

To validate the present sensor as a joint velocity and temperature probe, it needs both high enough velocities and strong temperature fluctuations. The experimental setup, sketched in figure 15, consists in positioning the cantilever in the wakes of a cylinder. The cylinder is a copper tube, with external diameter $\Phi=12 \mathrm{~mm}$. The mean velocity is $2.4 \mathrm{~m} / \mathrm{s}$. 


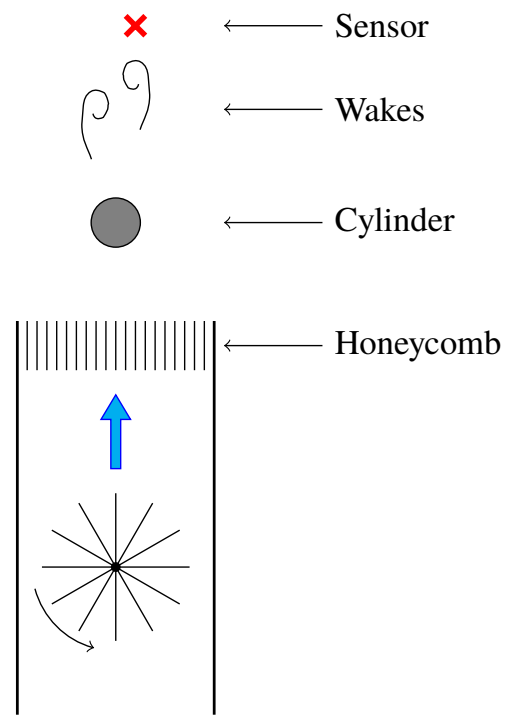

Figure 15: Sketch of experimental setup. The diameter of the cylinder is $\Phi=12 \mathrm{~mm}$.

The non-dimensional frequency of the vortex shedding, $f_{v}$, is the Strouhal number,

$$
S t=\frac{f_{v} \Phi}{\langle v\rangle} .
$$

The control parameter is the Reynolds number based on the cylinder diameter,

$$
R e_{\phi}=\frac{\Phi\langle v\rangle}{\nu}=1820 .
$$

In this range of Reynolds number, the vortex street is fully turbulent [50 and the Strouhal number is expected to be of order 0.2. Therefore, the shedding frequency, $f_{v}$, is expected to be

$$
f_{v}=\frac{S t\langle v\rangle}{\Phi}=40 \mathrm{~Hz} .
$$

Water can be circulated across the copper tube. The temperature regulation is achieved with a Lauda RP 855 chiller. The aim is to heat, or cool, the vortices shed by the cylinder. Indeed, these vortices form from the instability of the boundary layer. Thermal conduction inside the boundary layer can warm up or cool down the vortices, before they are periodically shed.

Velocity and temperature signals are obtained from the cantilever micro-system using the methods described in previous sections, a 4-wire measurement of the platinum resistor, and a lock-in measurement of the strain bridge imbalance, but with a lower sampling frequency of $200 \mathrm{~Hz}$, well suited to longer measurements. The spectra of velocity and temperature, and their cross-spectra are shown in figure 16. They have been computed with the Welch method, and window segment length of $2^{8}$ points. The temperature-velocity cross-spectrum, defined as,

$$
\begin{aligned}
P_{v T}=\int_{-\infty}^{\infty}\left[\int_{-\infty}^{\infty}(v(\tau)-\langle v\rangle) \times\right. \\
\quad(T(\tau+t)-\langle T\rangle) \mathrm{d} \tau] e^{-j \omega t} \mathrm{~d} t,
\end{aligned}
$$

is also computed with a Welch method with window segment length of $2^{8}$ points.

Measurements have been carried out with no coolant circulation and the copper cylinder at thermal equilibrium with the room (black curves), with water at $50{ }^{\circ} \mathrm{C}$ (red dashed curves), and with water at $5{ }^{\circ} \mathrm{C}$ (dot-dash blue curve).

The velocity power spectra are all fairly identical, as expected in these conditions where natural convection would be negligible. One peak is visible close to $20 \mathrm{~Hz}$, half the vortex-shedding frequency predicted by Eq. 30 The formation mechanism of this sub-harmonic peak has not been explored, because our motivation was only to produce 

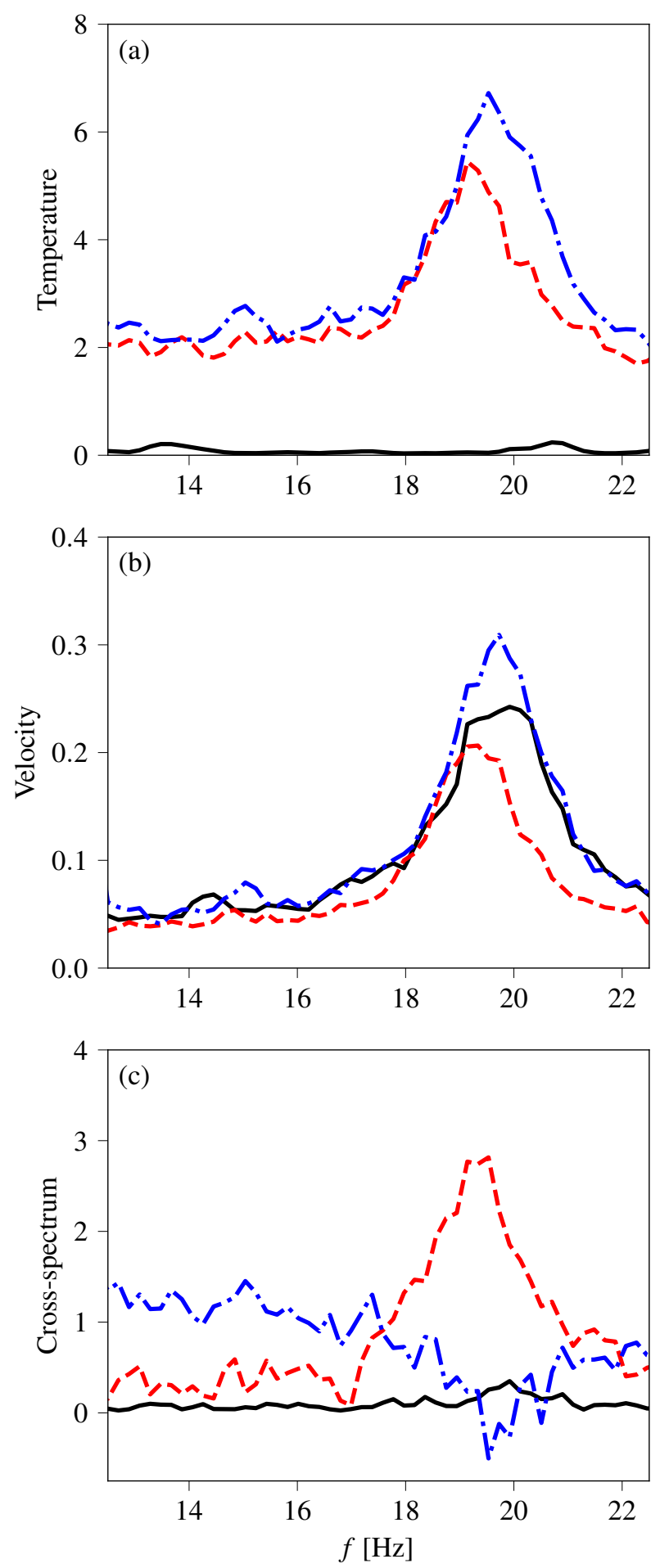

Figure 16: Signals obtained behind a cylinder. (a) Power spectra of the temperature signals, (b) power spectra of the velocity signals, (c) cross-spectra of temperature and velocity signals. Solid black line: isothermal cylinder. Dashed red line: warmed cylinder. Blue dot-dash line: cooled cylinder. 
a well-defined periodic coherent structure. Still, we can speculate that it arises from a coupling between the vortex shedding and finite size of the "jet-cylinder" configuration.

When the cylinder is isothermal, no peak can be found on the temperature spectrum and the signal has very few fluctuations. It is similar to the background noise of the system. When the cylinder is heated, or cooled, the spectrum exhibits a higher base value and a peak, at the frequency of the vortex shedding. Slight discrepancy between peak maxima are caused by the slight hysteresis of the turbine motor. There is little difference between heated or cooled cylinder on the temperature spectrum as power spectra are quadratic quantities. This is consistent with our interpretation of warm (or cold) eddies periodically crossing the sensor.

The velocity-temperature cross-spectrum, shown in figure $16 \mathrm{c}$, show a correlation at a frequency of order $f_{v}$ for heated cylinder, and anti-correlation at the same frequency for cooled cylinder. Indeed, when the cylinder is heated, warm coherent eddies are shed. When such a coherent structure crosses the sensor, it yields higher velocity and higher temperature signal. Conversely, when the cylinder is cooled, cold coherent eddies are shed, and they still yield higher velocity but lower temperatures. Of course, when the cylinder is isothermal, no cross-correlation is expected, and the experimental curve gets to the background noise.

\section{Conclusion and perspectives}

We have designed, and operated a prototype of fully micromachined joint temperature and velocity local sensor. The calibration and turbulent fluctuations in several types of flows validate the working principle: (i) the classical configuration of the turbulent round jet allows to validate the velocity signal, both at room temperature and cryogenic temperature; (ii) the Rayleigh-Bénard cell in the Barrel of Ilmenau allows to validate the temperature fluctuation signal in air; (iii) the correlation in the wakes of a heated, or cooled, cylinder provides direct evidence of accurate local cross-correlation measurements.

There are three main limitations of the current prototype: (i) the sensitivity of the strain bridge is too small to use the sensor in thermal convection in air; (ii) the platinum layer is unsuitable for temperature measurements in the cryogenic environment; (iii) the lack of electrical insulation makes the prototype unsuited to measurements in water.

All those limitations can be tackled for specific use-case: the sensitivity can be increased with a longer beam, at the cost of lowering the mechanical resonance frequency, which would be fine for applications to natural convection in air; dedicated materials such as niobium nitride can be used instead of platinum for low temperature applications [51]; and an additional protection layer can be added in the fabrication process to protect the conducting elements from electrical contact with water.

One advantage of the cantilever approach is that it can measure the velocity component in both directions. We have demonstrated that events of negative velocity can be detected inside a turbulent jet. This is important for its use as a local turbulent heat-flux sensor.

This work demonstrates the wide range of hydrodynamics applications that could benefit from dedicated cantilever-based sensors. One may think, for example, to measure the amount of heat transported by individual plumes in turbulent thermal convection, which would bring valuable experimental information in the field of turbulent Rayleigh-Bénard convection.

\section{Acknowledgments}

The sensor development was funded by LABEX iMUST (ANR-10-LABX-0064) of Universite de Lyon, within the program "Investissements d'Avenir" (ANR-11-IDEX-0007) operated by the French National Research Agency (ANR), and by "European High-performance Infrastructures in Turbulence" (EuHIT), European Grant Agreement no. 312778. We also thank support from SHREK collaboration and contract ANR-09-BLAN-0094-01.

The access to the Barrel of Ilmenau and CERN, and their operating costs, was funded by EuHIT Infrastructure Transnational Access Program. We would like to acknowledge the help and support of S. Abawi, V. Mitschunas and R. Kaiser, as well as O. Liot, at the Barrel. The CERN, the Technology Department and the Cryogenic Group CRG are warmly thanked for support and their hospitality.

This work was partly supported by the French RENATECH network and its FEMTO-ST technological facility. In particular, we warmly thank L. Robert, J.-Y. Rauch, D. Belharet, E. Herth, J. Valentin, V. Petrini, E. Courjon and J.-C. Jeannot for their help.

We acknowledge the work of K. Yaya, from IUT Saint-Étienne, who worked on the air jet, as part of her final year internship. 
We thank M. Tanase for his help with the probe support, and M. Moulin for the design and construction of mechanical components in ENS Lyon, and B. Van de Moortèle for his nice introduction to the subtleties of the scanning electron microscope and the EDS-X systems at ENS Lyon. We thank T. Crozes at Institut Néel for his help with the bonding machine.

\section{References}

[1] S. B. Pope, Turbulent flows (Cambridge University Press, 2000).

[2] G. Comte-Bellot, J. Phys. Colloques 37, C1 (1976).

[3] B. Castaing, Y. Gagne, and E. Hopfinger, Physica D 46, 177 (1990).

[4] R. Benzi, S. Ciliberto, R. Tripiccione, C. Baudet, F. Massaioli, and S. Succi, Phys. Rev. E 48, R29 (1993)

[5] P. Chainais, P. Abry, and J.-F. Pinton, Phys. fluids 11, 3524 (1999).

[6] U. Frisch, Turbulence: The Legacy of A. N. Kolmogorov (Cambridge University Press, 1996).

[7] D. Duri, C. Baudet, J.-P. Moro, P.-E. Roche, and P. Diribarne, Rev. Sci. Instrum. 86, 025007 (2015).

[8] S. C. C. Bailey, G. J. Kunkel, M. Hultmark, M. Vallikivi, J. P. Hill, K. A. Meyer, C. Tsay, C. B. Arnold, and A. J. Smits, J. Fluid Mech. 663, 160 (2010)

[9] Y. Borisenkov, G. Gulitski, M. Kholmyansky, S. Krylov, A. Liberzon, and A. Tsinober, J. Turbul. 16, 525 (2015).

[10] O. Chanal, B. Baguenard, O. Béthoux, and B. Chabaud, Rev. Sci. Instrum. 6, 2442 (1997).

[11] S. Pietropinto, C. Poulain, C. Baudet, B. Castaing, B. Chabaud, Y. Gagne, B. Hébral, Y. Ladam, P. Lebrun, O. Pirotte, and P. Roche, Physica C 386, 512 (2003).

[12] F. Moisy, O. Doaré, T. Pasutto, O. Daube, and M. Rabaud, J. Fluid Mech. 507, 175 (2004).

[13] K. J. Bullock and K. Bremhorst, IEEE Transactions on Instrumentation and Measurements 18, 163 (1969).

[14] H. Pabiou, J. Salort, C. Ménézo, and F. Chillà, Energy Procedia 78, 2911 (2015)

[15] K. Bremhorst, J. Phys. E: Sci. Instrum. 18, 44 (1985).

[16] S. Barth, H. Koch, A. Kittel, J. Peinke, J. Burgold, and H. Wurmus, Rev. Sci. Instrum. 76, 075110 (2005).

[17] J. Puczylowski, A. Hölling, J. Peinke, R. Bhiladvala, and M. Hölling, Meas. Sci. Technol. 26, 055302 (2015).

[18] J. Salort, A. Monfardini, and P.-E. Roche, Rev. Sci. Instrum. 83, 125002 (2012)

[19] B. K. Shivamoggi and R. A. Antonia, Fluid Dynamics Research 26, 95 (2000).

[20] Z. Warhaft, Annu. Rev. Fluid Mech. 32, 203 (2000).

[21] L. Pietri, M. Amielh, and F. Anselmet, Int. J Heat Fluid Flow 21, 22 (2000).

[22] O. Williams, T. V. Buren, and A. J. Smits, Exp. Fluids 56, 142 (2015)

[23] M. Munzel and A. Kittel, in Progress in Turbulence, Springer Proceedings in Physics, Vol. 101, edited by J. Peinke, A. Kittel, S. Barth, and M. Oberlack (2005) pp. 155-158.

[24] G. Arwatz, C. Bahri, A. J. Smits, and M. Hultmark, Meas. Sci. Technol. 24, 125301 (2013)

[25] R. du Puits, A. Loesch, J. Salort, and F. Chillà, Submitted to J. Fluid Mech. (2017).

[26] D. J. Tritton, Physical Fluid Dynamics 2nd Ed. (Oxford Science Publications, 1988).

[27] S. F. Hoerner, Fluid Dynamic Drag: practical information on aerodynamic drag and hydrodynamic resistance (Hoerner Fluid Dynamics, Brick Town, New Jersey, 1965). 
[28] L. D. Landau and E. M. Lifshitz, Theory of Elasticity (Pergamon, Oxford, 1970).

[29] J. Puczylowski, Sensor development for highly resolved measurements in turbulent flows, Ph.D. thesis, Der Fakultät für Mathematik und Naturwissenschaften der Carl von Ossietzky Universität Oldenburg (2015).

[30] J. E. Sader, J. Appl. Phys. 84, 64 (1998).

[31] S. Rast, C. Wattinger, U. Gysin, and E. Meyer, Rev. Sci. Instrum. 71, 2772 (2000).

[32] U. Gysin, S. Rast, P. Ruff, E. Meyer, D. W. Lee, P. Vettiger, and C. Gerber, Phys. Rev. B 69, 045403 (2004)

[33] L. Bellon, J. Appl. Phys. 104, 104906 (2008)

[34] F. A. Sandoval, M. Supúlveda, L. Bellon, and F. Melo, Sensors 15, 27905 (2015).

[35] D. Lohse and A. Müller-Groeling, Phys. Rev. Lett. 74, 1747 (1995).

[36] J. Mi and R. A. Antonia, Phys. Rev. E 64, 026302 (2001)

[37] J. Jiménez, J. Fluid Mech. 376, 139 (1998).

[38] A. Noullez, G. Wallace, W. Lempert, R. B. Miles, and U. Frisch, J. Fluid Mech. 339, 287 (1997).

[39] J. Jiménez, A. A. Wray, P. G. Saffman, and R. S. Rogallo, J. Fluid Mech. 255, 65 (1993).

[40] T. Gotoh, D. Fukayama, and T. Nakano, Phys. Fluids 14, 1065 (2002).

[41] K. R. Sreenivasan and B. Dhruva, Progress of Theoretical Physics Supplement, 103 (1998).

[42] H. Mouri and M. Takaoka, Phys. Rev. E 65, 056304 (2002).

[43] R. du Puits, C. Resagk, and A. Thess, New J. Phys. 15, 013040 (2013)

[44] O. Liot, J. Salort, R. Kaiser, R. du Puits, and F. Chillà, J. Fluid Mech. 786, 275 (2016).

[45] F. Chillà and J. Schumacher, Eur. Phys. J. E 35, 58 (2012).

[46] F. Gauthier, J. Salort, O. Bourgeois, J.-L. Garden, R. du Puits, A. Thess, and P.-E. Roche, EPL 87, 44006 (2009).

[47] A. Belmonte, A. Tilgner, and A. Libchaber, Phys. Rev. E 50, 269 (1994)

[48] J. Salort, O. Liot, E. Rusaouen, F. Seychelles, J.-C. Tisserand, M. Creyssels, B. Castaing, and F. Chillà, Phys. Fluids 26, 015112 (2014)

[49] S. Ciliberto and C. Laroche, Phys. Rev. Lett. 82, 3998 (1999)

[50] J. H. Lienhard, Research Division Bulletin, Washington State University 300 (1966).

[51] O. Bourgeois, E. Andre, C. Macovei, and J. Chaussy, Rev. Sci. Instrum. 77, 126108 (2006). 